\title{
Effect of pomegranate juice consumption on biochemical parameters and complete blood count
}

\author{
EIRINI MANTHOU ${ }^{1,2}$, KALLIOPI GEORGAKOULI ${ }^{1-3}$, CHARIKLIA K. DELI $^{1,2}$, \\ AGGELOS SOTIROPOULOS $^{1}$, IOANNIS G. FATOUROS ${ }^{1,2}$, DIMITRIOS KOURETAS ${ }^{4}$, SERKO HAROUTOUNIAN ${ }^{5}$, \\ CHRYSOULA MATTHAIOU ${ }^{5}$, YIANNIS KOUTEDAKIS ${ }^{1,2,6}$ and ATHANASIOS Z. JAMURTAS ${ }^{1,2}$
}

\author{
${ }^{1}$ Department of Physical Education and Sport Science, University of Thessaly; ${ }^{2}$ Department of Kinesiology, Institute for \\ Research and Technology Thessaly, Karies, Trikala 42100; ${ }^{3}$ Department of Nutrition and Dietetics, Technological Educational \\ Institute of Thessaly, Karditsa 43100; ${ }^{4}$ Department of Biochemistry and Biotechnology, University of Thessaly, \\ Larissa 41221; ${ }^{5}$ Laboratory of Nutritional Physiology and Feeding, Agricultural University of Athens, Athens 11855, Greece; \\ ${ }^{6}$ School of Sport, Performing Arts and Leisure, University of Wolverhampton, Walsall, West Midlands WS1 3BD, UK
}

Received June 9, 2016; Accepted January 20, 2017

DOI: $10.3892 /$ etm.2017.4690

\begin{abstract}
Pomegranate has been used therapeutically for centuries. The aim of the present study was to examine the effects of pomegranate juice (PJ) supplementation on complete blood count (CBC), glucose, blood lipids and C-reactive protein (CRP) in healthy subjects. A total of 5 males and 5 females (aged $31.8 \pm 6.6$ years, weighing $66.2 \pm 12.9 \mathrm{~kg}$ ) were randomly assigned into one of two groups and either consumed $500 \mathrm{ml}$ $\mathrm{PJ} /$ day or no PJ for 14 days. Blood samples were obtained from participants prior to and following the experimental period. PJ consumption resulted in a significant increase in red blood cell count $(\mathrm{P}<0.05)$, hemoglobin levels $(\mathrm{P}<0.001)$ and hematocrit levels $(\mathrm{P}<0.05)$. Other $\mathrm{CBC}$ parameters, glucose, cholesterol, triglycerides, high-density lipoprotein, low-density lipoprotein and CRP levels did not significantly change following PJ consumption. These results indicate that PJ intake for a short period of time may result in increased erythropoiesis or decreased degradation without any significant alterations in factors associated with metabolic health and inflammation in healthy individuals.
\end{abstract}

\section{Introduction}

Pomegranate is a fruit that has been cultivated for centuries and is considered of high nutritional and therapeutic value (1). Its biological and therapeutic properties are primarily attributed to the presence of polyphenols

Correspondence to: Professor Athanasios Z. Jamurtas, Department of Physical Education and Sport Science, University of Thessaly, Karies, Trikala 42100, Greece

E-mail: ajamurt@pe.uth.gr

Key words: red blood cells, hemoglobin, glucose, blood lipids, supplementation (ellagitanins, flavonoids, phenolic acids, stilbenes, tannins and anthocyanins) $(2,3)$, which are free radical scavenging compounds (4). Pomegranate is also rich in vitamins and minerals (5). Generally, pomegranate juice (PJ) is the greatest contributor to pomegranate ingestion in daily nutrition. Notably, the in vitro antioxidant activity of PJ has been determined to be threefold higher compared with red wine and green tea and is typically more potent than that of other natural juices $(6,7)$.

Based on phytochemical compounds and their activity in PJ, research on the effects of PJ supplementation on health parameters has provided promising results regarding oxidative stress, inflammation and metabolic diseases (8). These studies have been conducted in vitro (9), in animals (10) and humans $(11,12)$. Research conducted on atherosclerotic mice indicated that PJ supplementation reduced the size of atherosclerotic lesions and inhibited disease progression (13-15). Research on patients with cardiovascular disease demonstrated that following PJ supplementation, low density lipoprotein (LDL) levels were reduced $(16,17)$, stress induced ischemia (18) and vascular thickness decreased (19), while positive findings were particularly evident in patients at higher risk of coronary heart disease (20). In healthy individuals, PJ consumption reduced the potential for LDL aggregation, which represents an important step towards formation of foam cells and thus vascular thrombi (13). Furthermore, it has been indicated that PJ intake on a regular basis may affect cholesterol metabolism in macrophages, leading to reduced cellular uptake of oxidized LDL (12). PJ is also able to reduce nitric oxide synthase expression in endothelial cells of coronary vessels (15). In 2001, Das et al (21) examined the effect of pomegranate seed extract ingestion in diabetic rats. The results indicated that there was a significant decrease in glucose levels of $\sim 50 \%, 12 \mathrm{~h}$ after taking the extract, regardless of the dose given (300 and $600 \mathrm{mg} / \mathrm{kg}$ ). These findings may be the result of an increased absorption of glucose by peripheral tissues, which is associated with the presence of polyphenols in pomegranate and their function (22). 
Studies in humans and mice demonstrated that even moderate PJ consumption reduced susceptibility to free radical lipid peroxidation, while it increased resistance to LDL and high density lipoprotein (HDL)-cholesterol oxidation (23). Inhibition of lipid peroxidation with PJ supplementation has been verified in carotid artery stenosis patients (18), type II diabetics (12) and healthy individuals (3). In 2014, Matthaiou et al (3) also determined that glutathione (GSH) levels were increased following two weeks PJ supplementation, indicating that PJ induced beneficial changes in erythrocyte antioxidant concentration. In 2009, Boussetta et al (24) reported that punicic acid, a conjugated unsaturated fatty acid which is derived from the oil of pomegranate seeds, has anti-inflammatory action due to its potential to reduce the activity of neutrophils. Specific tannins in pomegranate also exhibited anti-inflammatory action in mice (25).

Although there have been numerous studies reporting the benefits of the polyphenols contained in PJ on health parameters in those with various diseases, research on healthy humans is scarce. The antioxidant properties of polyphenolic flavonoids may enhance cell resistance to oxidative stress, including red blood cells; however, to the best of our knowledge, the effects of PJ consumption on complete blood counts (CBC) have not been investigated. Therefore, the purpose of the present study was to examine the effects of PJ supplementation on $\mathrm{CBC}$, in addition to glucose, blood lipids and C-reactive protein (CRP) levels in healthy individuals.

\section{Materials and methods}

Participants. A total of 10 healthy and recreationally active individuals (5 males and 5 females) aged $31.8 \pm 6.6$ years and weighing $66.2 \pm 12.9 \mathrm{~kg}$ were recruited for the current study through flyers and word of mouth in the region of Thessaly, Greece. Power analysis was run using G*Power Data Analysis (Heinrich-Heine-University Düsseldorf, Düsseldorf, Germany) to estimate the required sample size. It was calculated that the necessary sample size was $\mathrm{n}=10$ for statistical power $0.8, \mathrm{P}<0.05$ and effect size 0.4. Although the majority of previous studies on PJ supplementation have used 14-18 subjects, the sample size of the present study is enough to support the findings. Inclusion criteria included the absence of any clinical symptom or chronic disease as determined by a health questionnaire. Exclusion criteria included the presence of gastrointestinal or metabolic disease, the taking of any medication (including nutritional supplements such as vitamins, minerals and phytosterols), smoking, current pregnancy or lactation in women or the following of a specific diet regime. Written informed consent was obtained from all participants when they had been informed of all risks, discomforts and benefits involved with the current study. The procedures were in accordance to the 1975 Helsinki declaration and were approved by the Institutional Review Board of the University of Thessaly (Larissa, Greece).

Study design. During the first visit, participants received information regarding the trial, completed a health and a physical activity questionnaire and signed the consent form. A randomized, counterbalanced, within-subjects experimental design was applied and participants were randomly assigned into two groups (each, $\mathrm{n}=5$ ). One group consumed $500 \mathrm{ml} /$ day
PJ for fourteen days (the experimental group); the other group consumed no PJ for fourteen days (the control group). The four experimental weeks were separated by a one-week washout period. All participants reported to the laboratory at the start and the end of each two-week phase. In each test day at the two-week time point, anthropometric and physiological measurements in addition to blood sampling took place from 8-10 a.m., following an overnight fast. Alcohol consumption and smoking were not allowed for $12 \mathrm{~h}$ prior to each test day. Water consumption was ad libitum. All measurements were performed under controlled environmental conditions (the room temperature was $22 \pm 2^{\circ} \mathrm{C}$, with a relative humidity of $45 \pm 4 \%$ ).

Anthropometric and physiological measurements. Body mass, body fat and hydration level were assessed using leg-to-leg bioelectrical impedance scale (Tanita BF 522 W; Tanita Europe BV, Amsterdam, The Netherlands) while participants were lightly dressed and barefooted. Standing height was measured to the nearest $0.1 \mathrm{~cm}$ (Stadiometer 208, Seca GmbH, Hamburg, Germany). Body mass index (BMI) was calculated using the equation $\mathrm{BMI}=$ weight $(\mathrm{kg}) /[\text { height }(\mathrm{m})]^{2}$. Waist and hip circumferences were obtained with measuring tape. Waist to hip ratio (WHR) was calculated using the equation WHR=waist circumference/hip circumference. Blood pressure (BP) was measured with a manual sphygmomanometer (FC-101 Aneroid Sphygmomanometer; Focal Corporation, Tokyo, Japan); the lowest of three readings was recorded. Baseline anthropometric and physiological characteristics of all participants are presented in Table I.

Diet and physical activity. To ensure that diet and/or exercise preceding the trials would not affect biochemical parameters measured in the current study, participants were instructed to refrain from excessive exercise, record meals and physical activity two days prior to the first trial, and follow the same diet and activities for two days prior to each subsequent trial. Each participant was provided with a written set of guidelines and record sheets for recording food intake and physical activity. Diet records were subsequently analyzed using the computerized nutritional analysis system Science Fit Diet 200A (Science Technologies, Athens, Greece; Table II). Participants were instructed to follow their usual habits during the experiment otherwise.

Test food. The juice tested was a commercially available product (CBH-VITOM S.A., Athens, Greece). PJ was provided to the participants in bottles containing $250 \mathrm{ml}$ each and the participants were instructed to consume one in the morning and one in the afternoon (a total of $500 \mathrm{ml}$ per day). Each $250 \mathrm{ml}$ product was prepared from 2.5 squeezed pomegranates. Nutritional values per $100 \mathrm{ml}$ and as percentages of the Recommended Daily Allowance are presented in Table III. The polyphenolic composition of the PJ was measured by Matthaiou et al (3), as the PJ was the same as the one used in the present study. Briefly, the total polyphenolic content was $405.00 \mathrm{mg} / \mathrm{l}$ of equivalent gallic acid and the total amount of flavonoid was $12.67 \mathrm{mg} / \mathrm{l}$ of equivalent quercetin. PJ also contained different classes of polyphenols as flavonoids, phenolic acids and stilbenes. The concentrations of the 
Table I. Baseline anthropometric and physiological characteristics of all participants before and after the experimental period.

\begin{tabular}{lcccc}
\hline & \multicolumn{2}{c}{ EG } & & \multicolumn{2}{c}{ CG } \\
\cline { 2 - 4 } Characteristic & Pre & Post & Pre & Post \\
\hline Weight, Kg & $66.5 \pm 13.0$ & $66.3 \pm 12.6$ & $66.3 \pm 12.9$ & $20.8 \pm 12.6$ \\
Body Fat, $\%$ & $21.5 \pm 3.3$ & $21.3 \pm 3.1$ & $20.4 \pm 4.0$ & $0.79 \pm 0.1$ \\
WHR & $0.80 \pm 0.1$ & $0.79 \pm 0.1$ & $0.80 \pm 0.1$ & $103.0 \pm 10.6$ \\
SBP, mmHg & $107.0 \pm 9.8$ & $106.5 \pm 8.5$ & $69.5 \pm 9.5$ & $67.5 \pm 8.6$ \\
DBP, mmHg & $70.5 \pm 6.4$ & $71.0 \pm 6.1$ & $78.1 \pm 9.1$ & $73.5 \pm 11.3$ \\
Heart rate, bpm & $75.3 \pm 10.3$ & $73.4 \pm 10.1$ & $54.6 \pm 2.0$ & $54.4 \pm 2.0$ \\
Hydration state, $\%$ & $54.3 \pm 1.3$ & $54.3 \pm 1.8$ & & \\
\hline
\end{tabular}

Data are presented as mean \pm standard deviation. EG, experimental group; CG, control group; WHR, waist to hip ratio; SBP, systolic blood pressure; DBP, diastolic blood pressure.

Table II. Nutritional analysis of the 2-day diet records of all participants, in the experimental and control groups.

\begin{tabular}{|c|c|c|c|c|}
\hline \multirow[b]{2}{*}{ Component } & \multicolumn{2}{|c|}{ EG } & \multicolumn{2}{|c|}{$\mathrm{CG}$} \\
\hline & Pre & Post & Pre & Post \\
\hline Energy, kcal & $1771.5 \pm 425.1$ & $1765.8 \pm 403.0$ & $1737.8 \pm 398.2$ & $1783.8 \pm 406.4$ \\
\hline Protein, $g$ & $74.3 \pm 24.1$ & $71.4 \pm 25.7$ & $73.8 \pm 25.7$ & $73.5 \pm 23.3$ \\
\hline Carbohydrate, $g$ & $194.2 \pm 59.6$ & $193.3 \pm 40.4$ & $199.1 \pm 68.4$ & $200.7 \pm 69.9$ \\
\hline Fat, g & $73.3 \pm 22.7$ & $75.8 \pm 18.1$ & $71.7 \pm 21.0$ & $75.1 \pm 24.1$ \\
\hline Cholesterol, mg & $246.9 \pm 143.1$ & $240.3 \pm 127.2$ & $250.9 \pm 148.1$ & $228.5 \pm 110.1$ \\
\hline Fiber, $g$ & $15.8 \pm 8.4$ & $16.3 \pm 8.4$ & $15.5 \pm 9.9$ & $15.8 \pm 10.2$ \\
\hline Vitamin A, IU & $4,264.5 \pm 2,193.9$ & $4,443.4 \pm 2,895.5$ & $4,513.9 \pm 2,917.1$ & $4,527.7 \pm 2,854.3$ \\
\hline Vitamin $\mathrm{B} 12, \mu \mathrm{g}$ & $3.5 \pm 1.5$ & $3.6 \pm 1.7$ & $3.4 \pm 2.1$ & $3.6 \pm 1.5$ \\
\hline Vitamin B6, mg & $1.8 \pm 0.5$ & $1.9 \pm 0.6$ & $1.7 \pm 0.7$ & $1.8 \pm 0.5$ \\
\hline Vitamin $\mathrm{C}, \mathrm{mg}$ & $79.3 \pm 36.4$ & $77.6 \pm 29.0$ & $76.6 \pm 33.6$ & $71.5 \pm 34.5$ \\
\hline Vitamin D, mg) & $114.1 \pm 81.3$ & $116.3 \pm 75.0$ & $105.9 \pm 69.7$ & $100.9 \pm 56.1$ \\
\hline Vitamin E, mg_RE & $8.7 \pm 10.5$ & $9.0 \pm 9.9$ & $8.6 \pm 11.0$ & $8.2 \pm 8.9$ \\
\hline Thiamine, mg & $2.7 \pm 1.4$ & $2.4 \pm 1.2$ & $2.2 \pm 1.4$ & $2.6 \pm 1.3$ \\
\hline Niacin, mg & $21.8 \pm 7.9$ & $21.0 \pm 8.3$ & $19.6 \pm 6.9$ & $21.1 \pm 6.8$ \\
\hline Pantothenic Acid, mg & $11.0 \pm 20.6$ & $10.2 \pm 17.3$ & $12.1 \pm 25.9$ & $10.3 \pm 19.1$ \\
\hline Riboflavin, mg & $2.4 \pm 1.9$ & $2.6 \pm 1.7$ & $2.3 \pm 1.6$ & $2.2 \pm 1.3$ \\
\hline Folate, $\mu \mathrm{g}$ & $351.6 \pm 106.4$ & $345.5 \pm 103.7$ & $346.8 \pm 138.8$ & $335.9 \pm 87.5$ \\
\hline Iron, mg & $14.0 \pm 3.2$ & $14.7 \pm 4.3$ & $14.7 \pm 4.6$ & $14.5 \pm 2.7$ \\
\hline Selenium, $\mu \mathrm{g}$ & $110.4 \pm 33.6$ & $105.0 \pm 40.5$ & $106.5 \pm 44.7$ & $103.4 \pm 39.4$ \\
\hline
\end{tabular}

Data are presented as mean \pm standard deviation. EG, experimental group; CG, control group.

polyphenols contained in the PJ used in the current study were comparable to those identified in previous studies (26). However, total polyphenols in PJ may vary significantly between different pomegranate cultivars (27).

Blood collection and analyses. Participants rested in the lab for $30 \mathrm{~min}$ prior to blood sample being obtained. Participants were in a seated position when $20 \mathrm{ml}$ blood was drawn from the forearm vein. A portion (1-2 ml) of the blood collected was used to determine the parameters of the $\mathrm{CBC}$ measured by an automatic hematology analyzer (Mythic ${ }^{\mathrm{TM}}$ 18; Orphee Medical SA, Geneva, Switzerland). Each measurement was performed twice. The remaining blood was centrifuged at $4^{\circ} \mathrm{C}, 1370 \times \mathrm{g}$ for $10 \mathrm{~min}$ to separate the plasma in a refrigerated centrifuge (Heraeus ${ }^{\mathrm{TM}}$ Biogigure Primo; Thermo Fisher Scientific, Inc., Waltham, MA, USA). Then the collected supernatant was transferred to Eppendorf tubes (Sarstedt AG \& Co., Numbrecht, Germany). The samples were stored at $-80^{\circ} \mathrm{C}$ and thawed only once prior to analysis. Glucose, total cholesterol, HDL-cholesterol and triglyceride concentrations in plasma 
Table III. Nutritional values of pomegranate juice per $100 \mathrm{ml}$ and as percentages of the RDA.

\begin{tabular}{lcc}
\hline Component & Per $100 \mathrm{ml}$ & \% RDA $(250 \mathrm{ml})$ \\
\hline Energy, kcal & 58 & 7 \\
Protein, g & 0.3 & 2 \\
Carbohydrate, g & 14.1 & 12 \\
Sugars, g & 11.6 & - \\
Fat, g & 0.3 & 1 \\
Saturated Fat, g & 0.0 & 0 \\
Fiber, g & 0.1 & 3 \\
Salt, mg & 0.01 & 1 \\
\hline
\end{tabular}

RDA, recommended daily allowance.

were determined using a biochemical analyzer (Clinical Chemistry Analyzer Z 1145; Zafiropoulos Diagnostica S.A., Koropi, Greece). LDL-cholesterol was calculated using the Friedewald equation (28). CRP was analyzed by a semi-quantitative method using a commercial available kit (CRP Latex Slide Test, Code 7300; Zafiropoulos Diagnostica S.A., Koropi, Greece) according to the principal of the latex agglutination assay described by Singer et al (29).

CBC test included: White blood cell (WBC), lymphocytes (LYM), monocytes (MON), granulocytes (GRA), lymphocyte percentage (LYM\%), monocyte percentage (MON\%), granulocyte percentage (GRA\%), red blood cell (RBC) count, hemoglobin (HGB), hematocrit (Hct), mean corpuscular volume (MCV), mean corpuscular hemoglobin $(\mathrm{MCH})$, mean corpuscular hemoglobin concentration (MCHC), red blood cell distribution width (RDW), platelets (PLT), mean platelet volume (MPV), plateletcrit (PCT) and platelet distribution width (PDW).

Statistical analysis. Analysis of data was performed with two-way (group x time) repeated measures analysis of variance. If a significant interaction was obtained, pairwise comparisons were performed through simple main-effect analysis using the Bonferroni test method. $\mathrm{P}<0.05$ was considered to indicate a significant difference. The statistical programme used was SPSS version 18 (SPSS Inc., Chicago, IL, USA). Results are presented as mean \pm standard deviation.

\section{Results}

General characteristics. Compliance with PJ consumption was $97.9 \%$. Anthropometric characteristics of participants did not change markedly during the 5-week experimental period. Analysis of the diet records did not reveal any significant difference in the macro and micronutrients of the groups prior to each blood sampling (Table II). No significant changes for group or group $\mathrm{x}$ time were identified for RBC count. However, there were significant differences with respect to time $(\mathrm{F} 1,18=4.42 ; \mathrm{P}<0.05)$. Pairwise comparison demonstrated a significant increase $(\mathrm{P}<0.05)$ in the $\mathrm{RBC}$ count following $\mathrm{PJ}$ supplementation, which did not occur in individuals in the control group (Fig. 1A).
$C B C$ parameters. No significant results for group or group $\mathrm{x}$ time were identified for hemoglobin. However, there were significant differences with respect to time $(\mathrm{F} 1,18=5.12$, $\mathrm{P}<0.05)$. Pairwise comparison indicated a significant increase $(\mathrm{P}<0.001)$ in the concentration of hemoglobin following PJ supplementation, which did not occur in individuals in the control group (Fig. 1B).

No significant results for group or group $\mathrm{x}$ time were identified for hematocrit. However, there were significant differences with respect to time $(\mathrm{F} 1,18=4.27, \mathrm{P}<0.05)$. Pairwise comparison indicated a significant increase $(\mathrm{P}<0.05)$ in hematocrit following PJ supplementation (Fig. 1C).

The remaining CBC parameters prior to and following completion of the two trials are presented in Table IV. In general, these parameters did not change in either group. A significant difference was observed for group $\mathrm{x}$ time $(\mathrm{F} 1,17=3.98, \mathrm{P}<0.05)$ in $\mathrm{MCHC}$ only. Pairwise comparison demonstrated a significant increase $(\mathrm{P}<0.05)$ in $\mathrm{MCHC}$ in the control group at the end of the experimental period (Table IV).

Biochemical parameters. Furthermore, PJ supplementation did not significantly alter the concentrations of total cholesterol, HDL, LDL, glucose and triglycerides (Table V). CRP values were not $>6 \mathrm{mg} / \mathrm{dl}$, indicating decreased inflammation either at baseline or following the experimental period.

\section{Discussion}

The results of the present study demonstrate that $\mathrm{PJ}$ consumption of $500 \mathrm{ml} /$ day for two weeks increased the RBC count, hemoglobin concentration and hematocrit in healthy individuals. Other parameters concerning complete blood count, metabolic health or inflammation were not altered in this cohort of individuals. Therefore, PJ intake for a short period of time may result in increased erythropoiesis or prevention of RBC degradation without any significant alterations in factors associated with metabolic health and inflammation in healthy individuals.

RBCs and hemoglobin constitute the transport system of oxygen from the lungs to the tissues. It has been demonstrated that a high concentration of polyphenols may increase the resistance of RBC to oxidative stress (30). Therefore, a potential explanation for the results of the current study is that the increased content of polyphenols in PJ may have prevented RBC destruction due to reduced oxidative stress. Indeed, this hypothesis is further supported by a study reporting higher reduced GSH levels in erythrocytes following $500 \mathrm{ml}$ PJ supplementation every day for two weeks (3). It was noted that this increase may be due to the induction of expression or the catalytic activity of enzymes involved in GSH biosynthesis that are known to be increased by plant polyphenols (31).

Similarly, the participants that consumed PJ exhibited a 3\% increase in hemoglobin by at the end of the two week intervention. This finding is particularly important considering the short period during which participants consumed the juice. It has been determined that flavonoids serve an important role in preventing oxidation of hemoglobin by various factors, such as hypochlorous acid (32). Flavonoids bind to hemoglobin and are able to inhibit oxidation of the hemoglobin molecule by oxidizing agents. In addition to flavonoids, PJ exhibits antioxidant activity 

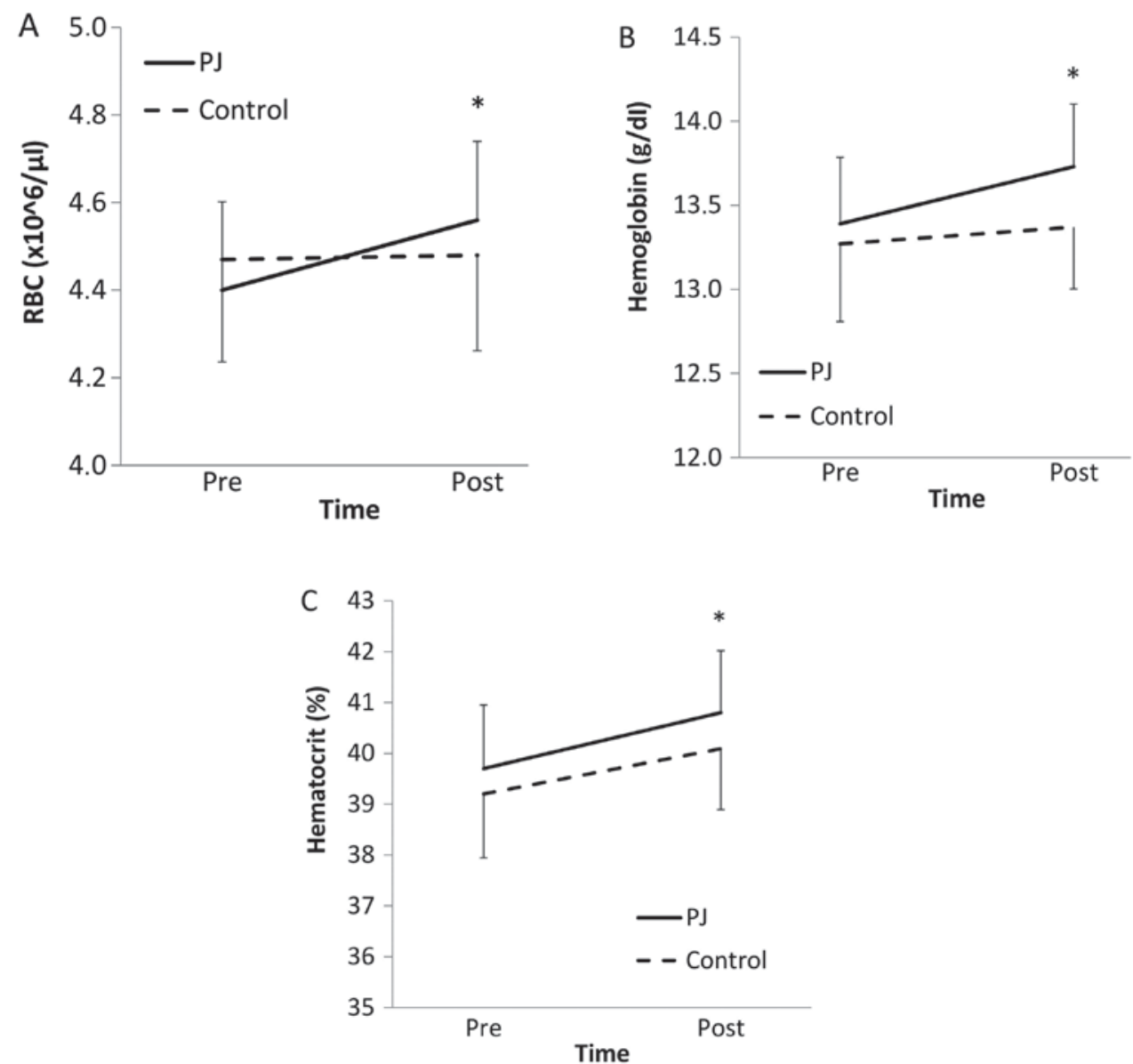

Figure 1. Changes in (A) RBC, (B) hemoglobin and (C) hematocrit following PJ consumption. ${ }^{*} \mathrm{P}<0.05$ vs. Pre of the same group. RBC, red blood cell; PJ, pomegranate juice.

that is much higher than that of red wine, green tea and other natural juices (4). Antioxidant capacity in erythrocytes has been identified to increase following two weeks of PJ supplementation in healthy individuals (3). Therefore, a potential explanation for the increased hemoglobin levels observed in the current study may be that the phytochemicals contained in PJ juice may have protected hemoglobin from oxidizing agents.

Hematocrit also exhibited a significant increase following two weeks of PJ supplementation. Increasing levels of hematocrit may be due to reduced RBC destruction. The high concentration of antioxidants in $\mathrm{PJ}$ is likely to have protected $\mathrm{RBC}$ and resulted in their subsequent reduced destruction. However, the measurements completed in the present study cannot confirm which factors caused this change. Therefore, further studies are required to highlight the contribution and importance of each factor, in addition to any potential synergistic effect.

Other parameters of the $\mathrm{CBC}$ did not change significantly over the course of PJ supplementation, with the exception of MCHC levels, which were significantly increased in the control group at the end of the experimental period. MCHC is an indicator of the average density of hemoglobin per RBC medium and usually increases in dehydration and hereditary spherocytosis, sickle cell disease and homozygous hemoglobin C disease (33). The change in MCHC was significant only for individuals in the control group. It may be suggested that the increase in MCHC levels is due to dehydration in the control group. However, assessment of hydration status performed through bioelectrical impedance (Table I) did not identify any significant differences over time or between groups. Indeed, this is something that requires further investigation.

Lipid profile was not altered following supplementation of PJ. Previous studies have reported that pomegranate may reduce blood lipid levels and lipid peroxidation $(3,34)$. In diabetic subjects with hyperlipidemia, consumption of PJ concentrate for 8 weeks significantly reduced levels of total cholesterol and LDL $(16,17)$. The absence of any significant changes in the present study may be due to the short duration of supplementation. Also, participants in the current study were individuals with normal lipid levels compared with previous studies where participants had elevated lipid levels $(15,16)$. A previous study has demonstrated that the higher the cardiovascular risk, the greater the changes occurring in risk factors such as cholesterol (20). Individuals in the current study were also normoglycaemic and CRP was within normal levels at baseline. Therefore, this may determine why changes in glucose and CRP were not evident in either group, although a decrease in both parameters has previously been recorded in rats following PJ supplementation $(21,24)$. Finally, studies investigating a greater number of participants would provide more reliable results on the effects of PJ supplementation in various health parameters.

PJ supplementation may result in an increased RBC count, hemoglobin concentration and hematocrit in healthy individuals. 
Table IV. Complete blood count parameters of all participants throughout the experimental period.

\begin{tabular}{|c|c|c|c|c|}
\hline \multirow[b]{2}{*}{ Parameter } & \multicolumn{2}{|c|}{ EG } & \multicolumn{2}{|c|}{$\mathrm{CG}$} \\
\hline & Pre & Post & Pre & Post \\
\hline $\mathrm{WBC}, \mathrm{x} 10^{3} / \mu \mathrm{l}$ & $5.9 \pm 0.8$ & $5.8 \pm 1.0$ & $6.0 \pm 0.7$ & $5.6 \pm 0.7$ \\
\hline LYM, \% & $38.0 \pm 7.6$ & $39.6 \pm 2.6$ & $38.4 \pm 6.0$ & $39.2 \pm 6.2$ \\
\hline MONO, \% & $9.6 \pm 2.0$ & $9.8 \pm 2.1$ & $11.3 \pm 4.3$ & $8.8 \pm 1.9$ \\
\hline GRA, $\%$ & $52.4 \pm 7.9$ & $50.6 \pm 2.3$ & $50.3 \pm 5.9$ & $51.9 \pm 5.6$ \\
\hline $\mathrm{MCV}, \mathrm{fl}$ & $88.6 \pm 7.6$ & $90.2 \pm 5.1$ & $88.8 \pm 7.8$ & $88.6 \pm 7.7$ \\
\hline $\mathrm{MCH}, \mathrm{pg}$ & $30.2 \pm 3.2$ & $30.7 \pm 2.3$ & $29.8 \pm 3.0$ & $30.5 \pm 3.0$ \\
\hline MCHC, g/dl & $33.9 \pm 0.9$ & $34.1 \pm 0.9$ & $33.5 \pm 0.9$ & $34.3 \pm 0.7^{\mathrm{a}}$ \\
\hline RDW, \% & $11.5 \pm 0.5$ & $11.5 \pm 0.9$ & $11.8 \pm 0.7$ & $11.3 \pm 0.6$ \\
\hline PLT, $\times 10^{3} / \mu 1$ & $254.2 \pm 84.5$ & $262.7 \pm 56.6$ & $247.9 \pm 65.6$ & $258.1 \pm 51.1$ \\
\hline MPV, fl & $8.2 \pm 0.9$ & $8.4 \pm 0.7$ & $8.6 \pm 0.9$ & $8.2 \pm 0.8$ \\
\hline PCT, $\%$ & $0.20 \pm 0.06$ & $0.22 \pm 0.03$ & $0.21 \pm 0.04$ & $0.21 \pm 0.03$ \\
\hline PDW, \% & $15.1 \pm 1.2$ & $14.8 \pm 1.7$ & $14.2 \pm 1.9$ & $15.1 \pm 1.2$ \\
\hline
\end{tabular}

${ }^{a} \mathrm{P}<0.05$ vs. Pre of the same group. Results are presented as mean \pm standard deviation. EG, experimental group; CG, control group; WBC, white blood cells; LYM, lymphocytes; MON, monocytes; GRA, granulocytes; MCV, mean corpuscular volume; MCH, mean corpuscular hemoglobin; MCHC, mean corpuscular hemoglobin concentration; RDW, red blood cell distribution width; PLT, platelets; MPV, mean platelet volume; PCT, plateletcrit; PDW, platelet distribution width.

Table V. Biochemical parameters of all participants in the experimental and control groups.

\begin{tabular}{lcccc}
\hline & \multicolumn{2}{c}{ Control } & \multicolumn{2}{c}{ Pomegranate } \\
\cline { 2 - 4 } Parameter, mmol/1 & Pre & Post & Pre & Post \\
\hline TG & $0.92 \pm 0.14$ & $0.87 \pm 0.12$ & $0.91 \pm 0.12$ & $0.95 \pm 0.14$ \\
TC & $4.9 \pm 0.3$ & $5.1 \pm 0.3$ & $5.0 \pm 0.3$ & $5.1 \pm 0.3$ \\
HDL & $1.51 \pm 0.09$ & $1.55 \pm 0.12$ & $1.53 \pm 0.11$ & $1.57 \pm 0.12$ \\
LDL & $2.98 \pm 0.31$ & $3.15 \pm 0.30$ & $3.07 \pm 0.31$ & $3.05 \pm 0.29$ \\
Glucose & $5.3 \pm 0.1$ & $5.2 \pm 0.1$ & $5.2 \pm 0.1$ & $5.1 \pm 0.1$
\end{tabular}

Data are presented as mean \pm standard deviation. TG, triglycerides; TC, total cholesterol; HDL, high density lipoprotein; LDL, low density lipoprotein.

Therefore, PJ supplementation may affect factors involved either in $\mathrm{RBC}$ formation and erythropoiesis, or in prevention of RBC degradation. It has been postulated that increased generation of ROS may contribute to the shorter lifespan of RBCs associated with iron-deficiency anemia (35). Therefore, antioxidants such as polyphenols may help counterbalance increased oxidative stress in RBCs, thus preventing their degradation. PJ is a rich source of polyphenols and nutrients and may therefore aid RBCs and other cells to counterbalance oxidative stress. Further research on this topic may reveal if $\mathrm{PJ}$ may be used as an adjunctive strategy for the prevention or treatment of certain types of anemia. In conclusion, two weeks of PJ supplementation resulted in an increased RBC count, hemoglobin concentration and hematocrit in healthy individuals. Due to the confirmed functional properties of PJ, further research is required to determine the long-term effects of PJ supplementation on healthy individuals in addition to the effect of different amounts of PJ on various biomedical parameters. Furthermore, it is necessary to investigate the effect of supplementation of PJ on factors affecting RBC formation and erythropoiesis, or prevention of RBC degradation.

\section{Acknowledgements}

The authors of the present study wish to thank CBH-VITOM S.A., Athens, Greece, for the provision of the commercial product of pomegranate juice used in the current study.

\section{References}

1. Vidal A, Fallarero A, Peña BR, Medina ME, Gra B, Rivera F, Gutierrez Y and Vuorela PM: Studies on the toxicity of Punica granatum L. (Punicaceae) whole fruit extracts. J Ethnopharmacol 89: 295-300, 2003.

2. Li Y, Guo C, Yang J, Wei J, Xu J and Cheng S: Evaluation of antioxidant properties of pomegranate peel extract in comparison with pomegranate pulp extract. Food Chem 96: 254-260, 2006. 
3. Matthaiou CM, Goutzourelas N, Stagos D, Sarafoglou E, Jamurtas A, Koulocheri SD, Haroutounian SA, Tsatsakis AM and Kouretas D: Pomegranate juice consumption increases GSH levels and reduces lipid and protein oxidation in human blood. Food Chem Toxicol 73: 1-6, 2014.

4. Gil MI, Tomás-Barberán FA, Hess-Pierce B, Holcroft DM and Kader AA: Antioxidant activity of pomegranate juice and its relationship with phenolic composition and processing. J Agric Food Chem 48: 4581-4589, 2000.

5. Mirdehghan SH and Rahemi M: Seasonal changes of mineral nutrients and phenolics in pomegranate (Punica granatum L.) fruit. Sci Hort 111: 120-127, 2007.

6. Castilla P, Dávalos A, Teruel JL, Cerrato F, Fernández-Lucas M, Merino JL, Sánchez-Martín CC, Ortuño J and Lasunción MA: Comparative effects of dietary supplementation with red grape juice and vitamin $\mathrm{E}$ on production of superoxide by circulating neutrophil NADPH oxidase in hemodialysis patients. Am J Clin Nutr 87: 1053-1061, 2008.

7. Seeram NP, Aviram M, Zhang Y, Henning SM, Feng L, Dreher M and Heber D: Comparison of antioxidant potency of commonly consumed polyphenol-rich beverages in the United States. J Agric Food Chem 56: 1415-1422, 2008.

8. Viuda-Martos M, Fernandez-López J and Pérez-Álvarez JA: Pomegranate and its many functional components as related to human health: A review. Compr Rev Food Sci Food Saf 9: 635-654, 2010.

9. Aharoni S, Lati Y, Aviram M and Fuhrman B: Pomegranate juice polyphenols induce a phenotypic switch in macrophage polarization favoring a M2 anti-inflammatory state. Biofactors 41: 44-51, 2015.

10. Faria A, Monteiro R, Mateus N, Azevedo I and Calhau C: Effect of pomegranate (Punica granatum) juice intake on hepatic oxidative stress. Eur J Nutr 46: 271-278, 2007.

11. Guo C, Wei J, Yang JJ, Xu J, Pang W and Jiang YG: Pomegranate juice is potentially better than apple juice in improving antioxidant function in elderly subjects. Nutr Res 28: 72-77, 2008.

12. Rosenblat M, Hayek T and Aviram M: Anti-oxidative effects of pomegranate juice (PJ) consumption by diabetic patients on serum and on macrophages. Atherosclerosis 187: 363-371, 2006.

13. Kaplan M, Hayek T, Raz A, Coleman R, Dornfeld L, Vaya J and Aviram M: Pomegranate juice supplementation to atherosclerotic mice reduces macrophage lipid peroxidation, cellular cholesterol accumulation and development of atherosclerosis. J Nutr 131: 2082-2089, 2001.

14. de Nigris F, Balestrieri ML, Williams-Ignarro S, D'Armiento FP, Fiorito C, Ignarro LJ and Napoli C: The influence of pomegranate fruit extract in comparison to regular pomegranate juice and seed oil on nitric oxide and arterial function in obese Zucker rats. Nitric Oxide 17: 50-54, 2007.

15. Esmaillzadeh A, TahbazF, Gaieni I, Alavi-Majd H and Azadbakht L: Concentrated pomegranate juice improves lipid profiles in diabetic patients with hyperlipidemia. J Med Food 7: 305-308, 2004.

16. Esmaillzadeh A, Tahbaz F, Gaieni I, Alavi-Majd H and Azadbakht L: Cholesterol-lowering effect of concentrated pomegranate juice consumption in type II diabetic patients with hyperlipidemia. Int J Vitam Nutr Res 76: 147-151, 2006.

17. Sumner MD,Elliott-Eller M, Weidner G, Daubenmier JJ, Chew MH, Marlin R, Raisin CJ and Ornish D: Effects of pomegranate juice consumption on myocardial perfusion in patients with coronary heart disease. Am J Cardiol 96: 810-814, 2005

18. Aviram M, Rosenblat M, Gaitini D, Nitecki S, Hoffman A Dornfeld L, Volkova N, Presser D, Attias J, Liker H and Hayek T: Pomegranate juice consumption for 3 years by patients with carotid artery stenosis reduces common carotid intima-media thickness, blood pressure and LDL oxidation. Clin Nutr 23: 423-433, 2004.
19. Davidson MH, Maki KC, Dicklin MR, Feinstein SB, Witchger M, Bell M, McGuire DK, Provost JC, Liker H and Aviram M: Effects of consumption of pomegranate juice on carotid intima-media thickness in men and women at moderate risk for coronary heart disease. Am J Cardiol 104: 936-942, 2009.

20. Fuhrman B, Volkova N and Aviram M: Pomegranate juice oxidized LDL uptake and cholesterol biosynthesis in macrophages. J Nutr Biochem 16: 570-576, 2005.

21. Das AK, Mandal SC, Banerjee SK, Sinha S, Saha BP and Pal M: Studies on the hypoglycaemic activity of Punica granatum seed in streptozotocin induced diabetic rats. Phytother Res 15 : 628-629, 2001.

22. Scalbert A, Manach C, Morand C, Rémésy C and Jiménez L: Dietary polyphenols and the prevention of diseases. Crit Rev Food Sci Nutr 45: 287-306, 2005.

23. Aviram M, Dornfeld L, Kaplan M, Coleman R, Gaitini D, Nitecki S, Hofman A, Rosenblat M, Volkova N, Presser D, et al: Pomegranate juice flavonoids inhibit low-density lipoprotein oxidation and cardiovascular diseases: Studies in atherosclerotic mice and in humans. Drugs Exp Clin Res 28: 49-62, 2002.

24. Boussetta T, Raad H, Letteron P, Gougerot-Pocidalo MA, Marie JC, Driss F and El-Benna J: Punicic acid, a conjugated linolenic acid, inhibits TNF $\alpha$-induced neutrophil hyperactivation and protects from experimental colon inflammation in rats. PLoS One 4: 6458, 2009.

25. Lee CJ, Chen LG, Liang WLand Wanga CC: Anti-inflammatory effects of Punica granatum Linne in vitro and in vivo. Food Chemistry 118: 315-322, 2012.

26. Elfalleh W, Tlili N, Nasri N, Yahia Y, Hannachi H, Chaira H, Ying $\mathrm{M}$ and Ferchichi A: Antioxidant capacities of phenolic compounds and tocopherols from Tunisian pomegranate (Punica granatum) fruits. J Food Sci 76: C707-C713, 2011

27. Hmid I, Elothmani D, Hanine H, Oukablic A and Mehinagic E: Comparative study of phenolic compounds and their antioxidant attributes of eighteen pomegranate (Punica granatum L.) cultivars grown in Morocco. Arab J Chem: doi: http://dx.doi. org/10.1016/j.arabjc.2013.10.011, 2013.

28. Friedewald WT, Levy RI and Fredrickson DS: Estimation of the concentration of low-density lipoprotein cholesterol in plasma, without use of the preparative ultracentrifuge. Clin Chem 18: 499-502, 1972.

29. Singer JM, Piotz CM, Parker E and Elster SF: The latex fixation test. III. Agglutination test for C-reactive proteins and comparison with the capillary precipitin method. Am J Clin Path 28: 611-617, 1957.

30. Youdim KA, Shukitt-Hale B, MacKinnon S, Kalt W and Joseph JA: Polyphenolics enhance red blood cell resistance to oxidative stress: In vitro and in vivo. Biochim Biophys Acta 1523: 117-122, 2000

31. Moskaug JØ, Carlsen H, Myhrstad MC and Blomhoff R: Polyphenols and glutathione synthesis regulation. Am J Clin Nutr 81 (1 Suppl): 277S-283S, 2005.

32. Gebicka L and Banasiak E: Hypochlorous acid-induced heme damage of hemoglobin and its inhibition by flavonoids. Toxicol In Vitro 26: 924-929, 2012.

33. Cohen ML and Rifkind D (eds): The Pediatric Abacus: Review of Clinical Formulas and How to Use Them. Parthenon Pub. Group, New York, NY, 2002.

34. Basu A and Penugonda K: Pomegranate juice: A heart-healthy fruit juice. Nutr Rev 67: 49-56, 2009.

35. Nagababu E, Gulyani S, Earley CJ, Cutler RG, Mattson MP and Rifkind JM: Iron-deficiency anemia enhances red blood cell oxidative stress. Free Radic Res 42: 824-829, 2008. 